\title{
Generación de señales para sistemas de radio sobre fibra basados en la combinación eléctrica de componentes de banda base y radiofrecuencia
}

\author{
Generation of signals for radio over fiber system based on electrical \\ combination of baseband and radiofrequency components
}

\author{
Manuel Cely ${ }^{1 *} \quad$ Rafael Muñoz ${ }^{1} \quad$ Gustavo Puerto $^{1} \quad$ Carlos Suárez $^{1}$
}

Recibido 25 de agosto de 2014, aceptado 6 de noviembre de 2015

Received: August 25, 2014 Accepted: November 6, 2015

\begin{abstract}
RESUMEN
En este artículo se describe la generación de señales para sistemas de radio sobre fibra usando combinación eléctrica de señales de banda base y radiofrecuencia. En estos sistemas las portadoras de radiofrecuencia se transportan como subportadoras de canal óptico sobre un enlace de fibra óptica en conexiones punto-punto o punto-multipunto. Los sistemas de radio sobre fibra se consideran como la piedra angular, bajo la cual se sustentarán las redes de nueva generación, al permitir que una única plataforma física soporte la transmisión de información proveniente de usuarios fijos y móviles. El artículo describe analíticamente el proceso de generación de señales mediante la definición del campo eléctrico en función de los índices de modulación de las señales de banda base y radiofrecuencia. Mediante modelado de la función de campo se encuentra que los índices de modulación presentan una fuerte dependencia entre sí y se obtiene su valor óptimo a fin de garantizar una buena calidad para ambas señales a la salida del transmisor.
\end{abstract}

Palabras clave: Fotónica de microondas, índice de modulación, modulador interferométrico modulación óptica, radio sobre fibra.

\begin{abstract}
This paper describes the generation of signals for radio over fibre system based on electrical combination of baseband and radiofrequency components. In these systems, the radio frequency carriers are conveyed as optical subcarriers over an optic fiber link in a point-to-point or point-tomultipoint connectivity paradigm. Radio over fiber systems are considered as the cornerstone over which next generation networks will be founded by allowing a single physical platform to supports the information exchange from both fixed and mobile users. The paper analytically describes the signal generation process by defining the electrical field as a function of the baseband and radiofrequency signals modulation depth. By means of modeling an electrical field, it was found a trade-off between the modulation depths of both signals; as well as the their optimum value in order to assure a good signal a the transmitter output.
\end{abstract}

Keywords: Microwave photonics, modulation depth, interferometer modulator, optical modulation, radio over fibre.

1 Universidad Distrital Francisco José de Caldas. Facultad de Ingeniería. Carrera 7 No 40B - 53. Bogotá, Colombia. E-mail:macelym@correo.udistrital.edu.co; romunozo@correo.udistrital.edu.co; gapuerto@udistrital.edu.co; csuarezf@udistrital.edu.co

* Autor de correspondencia 


\section{INTRODUCCIÓN}

La demanda de altas tasas de transmisión y gran ancho de banda en redes fijas e inalámbricas se ha incrementado en los últimos años y se pronostica un comportamiento similar en los años venideros. El fabricante de equipos de telecomunicaciones Cisco Systems publicó un reporte, el que con base en mediciones del tráfico actual de Internet, pronostica el tráfico de datos a nivel mundial hasta el 2018 [1]. El reporte especifica que el tráfico IP global anual superará el umbral del zettabyte alcanzando los 1,4 zettabytes a finales de 2017. El tráfico IP global se ha cuadruplicado en los últimos 5 años, y se triplicará en los próximos 5 años. En general, el tráfico IP crecerá a una tasa anual compuesta del 23\% entre 2012 hasta 2017. El tráfico en el segmento metropolitano superó el de la red de transporte en 2014 y representará el 58\% del tráfico IP total en 2017. Entre 2012 y 2017 en este segmento de red, el tráfico crecerá casi dos veces más que el tráfico de la red troncal. Del mismo modo se pronostica que para 2017 casi la mitad de todo el tráfico IP se originará en dispositivos que no son computadores personales. En 2012 solo el 26\% del tráfico IP de consumo se originó en dispositivos no-PC, pero para 2017 la proporción de tráfico IP no-PC crecerá hasta un $49 \%$. El tráfico originado en computadores personales crecerá a una tasa compuesta anual de $14 \%$, mientras que el tráfico originado en tablets, teléfonos móviles, televisores y dispositivos de comunicación máquina-máquina (M2M) tendrán tasas de crecimiento del tráfico generado del 104\%, $79 \%, 24 \%$ y $82 \%$, respectivamente. El tráfico de los dispositivos inalámbricos y móviles superará el de dispositivos cableados en 2016. En 2017 los dispositivos conectados por cable representarán el $45 \%$ del tráfico IP, mientras que WiFi y dispositivos móviles representarán el 55\% del tráfico IP. Así a corto plazo se prevé un gran aumento del ancho de banda originado desde dispositivos móviles. Hoy en día las redes de acceso ópticas pueden proporcionar gran ancho de banda a usuarios fijos. Por otro lado, las redes inalámbricas ofrecen una movilidad deseable a los usuarios, pero no cumplen con los requerimientos de ancho de banda. Además, el tener redes de acceso separadas genera un alto costo en términos de operación y mantenimiento. Todo esto sugiere la integración de dichas redes en una sola infraestructura compartida para la futura distribución de contenidos a usuarios fijos y móviles. En este contexto, los sistemas de Radio sobre Fibra (RoF) y fibra hasta el hogar (FTTH) son dos candidatos serios para consolidarse en el segmento de redes de acceso inalámbrico y fijo respectivamente, debido al gran ancho de banda que soportan. Aunque los sistemas RoF se pueden implementar en redes troncales cubriendo largas distancias [2], el mayor punto de atracción radica en el despliegue de sistemas RoF en redes de acceso inalámbrico de banda ancha que permitan el transporte y la distribución de las portadoras de RF de cualquier red inalámbrica utilizando una infraestructura de red FTTH. Mientras que la atenuación en espacio libre de frecuencias en la banda de microondas puede llegar a ser mayor de $10 \mathrm{~dB} / \mathrm{km}$, en fibra óptica estándar monomodo, la atenuación es de $0,2 \mathrm{~dB} / \mathrm{km}$ independientemente de la banda de frecuencia que se transporte. Este hecho, sumado al gran ancho de banda ofrecido por la fibra óptica, constituyen las principales razones por las cuales son atractivas las tecnologías de transmisión RoF. En este escenario la principal preocupación y, a la vez, el desafío se traduce en cómo transmitir las señales de banda base de las redes FTTH y de radiofrecuencia (RF) de las redes inalámbricas en una sola longitud de onda sobre una sola fibra de una manera costo-efectiva y con una calidad aceptable. A la fecha se han realizado diferentes trabajos que demuestran las ventajas de implementar sistemas de transporte de señales de radiofrecuencia sobre enlaces de fibra óptica en diferentes escenarios y aplicaciones. En [3] se discuten las tecnologías habilitantes que permiten el desarrollo de sistemas RoF que incluye esquemas para generación óptica de ondas milimétricas y elevadores de frecuencia, en [4] se presentan varias técnicas para la implementación de redes de acceso ópticas-inalámbricas basadas en procesos de modulación externa realizada en un modulador tipo Mach-Zehnder (MZ). Por otro lado, la modulación simultánea de señales en banda base y RF en un modulador óptico se demostró experimentalmente en [5]. En el contexto de estudios sobre las limitaciones en las prestaciones y desempeño en la transmisión de señales analógicas en enlaces de fibra, en [6] se presentó el efecto de intermodulaciones en banda y fuera de banda de canales multiplexados en longitud de onda (WDM) en una red de transporte RoF. Asimismo, se han reportado estudios sobre los efectos de las características no-lineales de diodos láser configurados en modulación directa [7] y 
efectos de distorsión causados por moduladores tipo MZ en enlaces RoF [8-9]. Posteriormente en [10-11] se definieron parámetros para identificar el máximo rango dinámico y las mínimas pérdidas de inserción en un enlace óptico modulado externamente con subportadoras de RF y en [12] se ampliaron los estudios mencionados anteriormente con el reporte y demostración de un análisis de prestaciones de calidad de un sistema de transmisión RoF considerando requerimientos generados por efectos de propagación de ondas milimétricas además de la evaluación de diferentes formatos de modulación. Asimismo, en [13] se presenta un estudio sobre los efectos de ruido e intermodulación en enlaces RoF que permiten identificar las limitaciones en el desempeño del sistema, también un análisis de la relación portadora-banda lateral [14], así como estrategias para reducir la figura de ruido en enlaces de fibra óptica modulados con señales analógicas [15] y un análisis sobre la optimización del punto de cuadratura de un modulador óptico tipo MZ para aplicaciones RoF [16]. Recientemente se publicó un estudio sobre el análisis de moduladores ópticos tipo MZ y de electroabsorción para aplicaciones de RoF y comunicaciones ópticas de espacio libre [17].

En este artículo se describe la generación de señales para sistemas RoF empleando combinación eléctrica de las componentes de banda base y radiofrecuencia. Se presenta un modelo analítico a partir del cual se obtiene la expresión de campo eléctrico en función de los índices de modulación y se modela dicha función con el fin de evaluar las prestaciones del sistema generador RoF y encontrar un punto óptimo de operación. Para ello se considera que la señal de entrada al modulador MZ es la combinación directa en el dominio eléctrico de la señal de RF y cualquier señal en banda base transportada en la portadora óptica.

\section{MODULACIÓN ELECTROÓPTICA}

Los mecanismos de modulación óptica se basan en alterar alguno de los parámetros de una señal óptica de forma proporcional a una segunda señal eléctrica. El efecto de la señal moduladora sobre la señal modulada se evalúa de forma cuantitativa mediante el índice de modulación, cuanto mayor es dicho índice, mayor es la variación del parámetro modificado en la señal portadora para la misma señal moduladora. La señal óptica generada por un láser se puede modular de forma directa mediante la variación de la corriente inyectada al dispositivo, o externamente, haciendo pasar el haz de luz emitido a través de un elemento controlable que cambia la amplitud y/o la fase de la luz. En este contexto se pueden considerar láseres directamente modulados y láseres modulados externamente. El presente estudio está basado en el paradigma de modulación externa en moduladores tipo Mach-Zehnder (MZ), los cuales se basan en el efecto electroóptico lineal o efecto Pockels [18]. Un modulador tipo MZ utiliza una estructura interferométrica implementada sobre un sustrato de Niobato de Litio $\left(\mathrm{LiNbO}_{3}\right)$, como se muestra en la Figura 1. En este dispositivo, la luz se divide en partes iguales entre dos guías de ondas paralelas en la superficie del sustrato y se recombina a continuación en la salida. La forma en que la variación en el índice de refracción se traduce en un cambio de alguna de las propiedades de la señal óptica: amplitud, frecuencia, fase o polarización, depende de la configuración del dispositivo; en particular, de las corrientes de polarización que controlan el elemento modulador y que tienen incidencia directa en los índices de modulación, relación de extinción y potencia de la señal modulada. En este contexto, una tensión de control (V) aplicada a los electrodos que se muestran en la figura genera un cambio en el índice de refracción a través del efecto electroóptico. Esto provoca un cambio en la constante de propagación y un desplazamiento de fase relativo en el punto donde las dos ondas se combinan. Cuando no hay tensión, el desplazamiento de fase relativa es cero y la señal recombinada sale del dispositivo sin atenuación (a excepción de las pérdidas en las guías de ondas). Cuando se aplica una tensión que produce un desplazamiento de fase de $\pi(V \pi)$ entre los dos brazos, la señal se extingue, por lo tanto, el dispositivo actúa como un interruptor controlado por tensión. Estos dispositivos funcionan a velocidades de hasta $50 \mathrm{~Gb} / \mathrm{s}$ [19]. Finalmente, $\mathrm{V} \pi$ es un parámetro propio de cada modulador.

Un modulador tipo MZ también permite la aplicación de voltajes de control en ambas guías de onda, lo que resulta en un dispositivo modulador externo de doble control. Esta característica permite la generación de señales en cuadraturas arbitrarias [20] que encuentran aplicación en procedimientos de transmisión, como modulación de portadora óptica suprimida. Los moduladores externos también se pueden utilizar para la modulación de fase en sistemas coherentes usando una única guía de onda con un índice de 
refracción modulado electroópticamente. Así pues, la modulación externa ofrece ventajas sobre la modulación directa del diodo láser en sistemas de fibra óptica, principalmente en términos de ancho de banda y rangos de linealidad. Este aspecto impulsó el desarrollo de tecnologías de fabricación de los moduladores ópticos integrados a un nivel actual de madurez muy alto [21-22]. La Figura 2 muestra la curva de transferencia de un modulador tipo MZ. Esta curva representa la transferencia de potencia óptica del dispositivo, en función del desfase electroóptico inducido sobre la señal óptica.

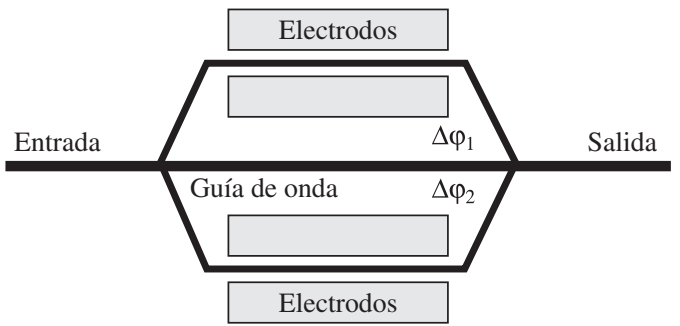

Figura 1. Modulador MZ de control doble.

Este desfase depende a su vez de la tensión de polarización aplicada sobre los electrodos. Como se puede observar existe una región donde la función de transferencia tiene carácter lineal y, que por consiguiente, resulta óptima para la modulación de la señal eléctrica sobre la portadora óptica. El dispositivo trabaja en régimen lineal cuando se aplica una tensión de polarización tal que se induce un desfase sobre la señal óptica igual a $\pi / 2$, y además los niveles de tensión aplicados son lo suficientemente pequeños para no distorsionar la señal de información. Las ecuaciones de modelado del modulador MZ tienen como finalidad obtener la expresión del campo eléctrico de la señal óptica a la salida del dispositivo, en función de las diferentes señales de entrada y de algunos parámetros del mismo. Como punto de partida, se formula una primera aproximación que proporciona el campo eléctrico a la salida del dispositivo en función del campo eléctrico a la entrada y de los desfases inducidos por las señales eléctricas aplicadas sobre los electrodos del modulador MZ como consecuencia del efecto electroóptico. La ecuación de campo se establece a partir de la geometría del modulador. En el dispositivo mostrado en la Figura 1, se aplica una señal eléctrica sobre uno de los dos brazos del interferómetro. Esta señal provoca mediante el efecto electroóptico un cambio de fase sobre la señal óptica que se propaga por dicho brazo [23]. La función de transferencia se puede expresar en términos del coeficiente de acoplo de la propagación del campo y el desfase producido en la señal en ambas ramas del interferómetro de la siguiente forma [13]:

$E_{\text {out }}(t)=\left(\frac{1}{\sqrt{2}}\right) E_{\text {in }}(t) \frac{\left[A_{1} e^{\left(j \Delta \phi_{1}\right)}+A_{2} e^{\left(j \Delta \phi_{2}\right)}\right]}{\alpha}$

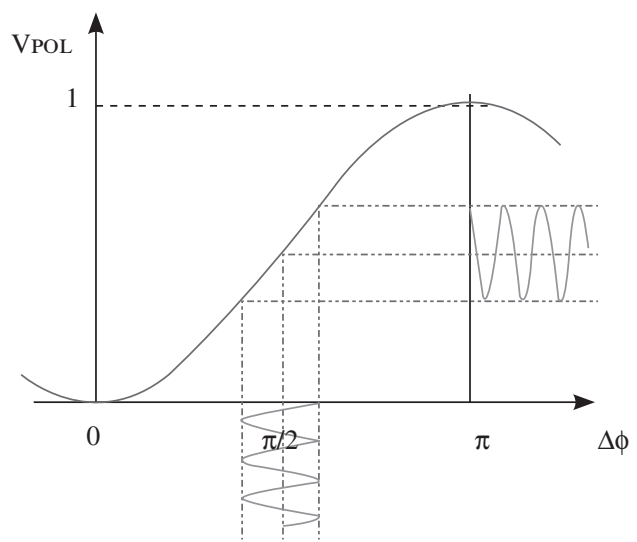

Figura 2. Curva de transferencia de potencia de un modulador MZ.

Donde $\alpha$ representa la atenuación de la señal a su paso por el dispositivo y $\mathrm{A}_{1}$ y $\mathrm{A}_{2}$ representan los coeficientes de acoplo de las ramas superior $\mathrm{e}$ inferior respectivamente en el modelo del modulador de control doble mostrado en la Figura $1, \varphi_{1}, \varphi_{2}$ representa los desfases en cada una de las ramas debido al efecto electroóptico. Estas variables se pueden expresar de la siguiente forma:

$$
\begin{gathered}
A_{1}=\alpha=\sqrt{0.5+\varepsilon} \\
A_{2}=\sqrt{1-\alpha^{2}} \\
\Delta \varphi_{1}=\frac{\pi}{V_{\pi(R F)}} V_{1(R F)}(t)+\frac{\pi}{V_{\pi(d c)}} V_{1(d c)} \\
\Delta \varphi_{2}=\frac{\pi}{V_{\pi(R F)}} V_{2(R F)}(t)+\frac{\pi}{V_{\pi(d c)}} V_{2(d c)}
\end{gathered}
$$

El término $\varepsilon$ en (2) representa la diferencia entre los coeficientes de acoplo de propagación de energía de la rama superior e inferior. Para un modulador MZ ideal, $\varepsilon=0$, lo cual indica que la potencia es dividida en partes iguales en las dos ramas. A su vez, 
los términos de variación de fase $\Delta \varphi$ dependen de la tensión de polarización $\mathrm{V}_{(\mathrm{dc})}$, este valor se define como aquella tensión que aplicada sobre los electrodos del dispositivo provoca un cambio de fase de $180^{\circ}$ sobre la señal óptica que se propaga por la guía de onda como consecuencia del efecto electroóptico. Valores típicos de tensiones de polarización para

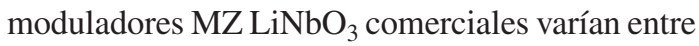
$4 \mathrm{~V}$ y $6 \mathrm{~V}$. Del mismo modo depende de $\mathrm{V}_{\mathrm{RF}}$, el cual representa el voltaje necesario en las entradas de $\mathrm{RF}$ (superior e inferior) para provocar un cambio de fase de $180^{\circ}$ entre los dos brazos del interferómetro. Si se asume un coeficiente de acoplo direccional en el cual el campo se distribuye uniformemente en los dos brazos, y $\alpha=2$ (para unas pérdidas de inserción típicas de un modulador MZ de $6 \mathrm{~dB}$ ), la ecuación (1) se puede expresar como:

$E_{\text {out }}(t)=\left(\frac{1}{\sqrt{2}}\right) E_{\text {in }}(t) \frac{\left[\left(\frac{1}{\sqrt{2}}\right) e^{\left(j \Delta \varphi_{1}\right)}+\left(\frac{1}{\sqrt{2}}\right) e^{\left( \pm j \Delta \varphi_{2}\right)}\right]}{2}$

Simplificando se obtiene la expresión general del campo eléctrico a la salida del modulador MZ:

$$
E_{\text {out }}(t)=\left(\frac{1}{4}\right) E_{\text {in }}(t)\left[e^{\left(j \Delta \varphi_{1}\right)}+e^{\left( \pm j \Delta \varphi_{2}\right)}\right]
$$

Finalmente, la expresión de una señal en un sistema radio sobre fibra se puede definir mediante:

$$
V_{R F}(t)=c(t)+e(t) \cos \left(\omega_{e} t+\alpha_{e}\right)
$$

Donde $c(t)$ es cualquier señal en banda base con cierta velocidad binaria transportada en la portadora óptica y $e(t)$ es la señal de datos que modula la portadora de RF definida por $\left(\omega_{\mathrm{e}}=2 \pi f_{\mathrm{e}}\right)$ más una constante de fase $\alpha_{e}$. Cabe resaltar que la modulación óptica es un proceso sensible a la polarización, en este trabajo se asumió un control de estado de polarización a la entrada del modulador. Así, el índice de modulación (IM) se define a través de la relación entre la amplitud de la señal moduladora y la tensión de desplazamiento de fase $\mathrm{V}_{\pi}$.

$$
I M_{\text {señal }}=\frac{V_{\text {señal }}}{V \pi}
$$

\section{GENERACIÓN DE SEÑALES PARA SISTEMAS RoF}

La combinación directa de la señal de RF multiplexada en subportadora es el método más directo para la generación de señales en un sistema de radio sobre fibra. De forma directa las señales en banda base y RF se mezclan en el dominio eléctrico usando un mezclador de microondas. Posteriormente, la señal combinada se convierte al dominio óptico mediante un modulador MZ de control sencillo. Las señales de banda base y RF utilizadas tienen la forma:

$$
\begin{gathered}
c(t)=A_{1} V_{B B}(t)+V_{d c B B} \\
e(t)=A_{2} V_{D R F}(t)
\end{gathered}
$$

En donde $c(t)$ está conformada por una señal de información de banda base $V_{B B}(t)$ de amplitud $A_{1}$ y un voltaje DC $V_{d c B B}$. Por otro lado, la señal de radiofrecuencia $e(t)$ está conformada por una señal de datos $V_{D R F}(t)$, con amplitud $A_{2}$. En este trabajo se usó una señal $e(t)$ con tasa de transmisión de 2.5 Gbps en una portadora de $10 \mathrm{GHz}$ y fase de oscilación nula. La señal $c(t)$ utilizada tiene una tasa de transmisión de 625 Mbps. La Figura 3 muestra la configuración para este esquema:

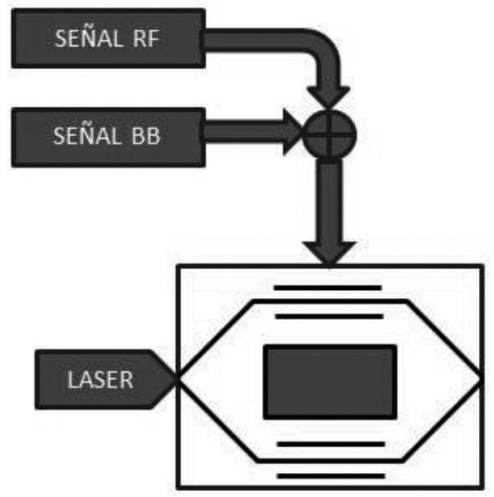

Figura 3. Esquema de modulación externa de un solo brazo con MZ

Para el esquema de la Figura 3, debido a que la propuesta solo se utiliza un brazo del modulador, la ecuación que describe el campo de salida es:

$$
E_{\text {out }}(t)=\frac{E_{\text {in }}}{4} \cos \left(\frac{\Delta \varphi}{2}\right) e^{\frac{j \Delta \varphi}{2}}
$$

Donde $\Delta \varphi=\Delta \varphi_{1}$.

La señal de información que se modula ópticamente está compuesta por las señales de banda base y de radiofrecuencia definidas en (8), al sustituirla en (4) y reemplazando en (12) se obtiene el campo eléctrico a la salida del modulador de la siguiente forma: 


$$
E_{\text {out }}(t)=\frac{E_{\text {in }}}{2} \cos \left(\begin{array}{l}
\frac{\pi}{2 V_{\pi(R F)}} \\
{\left[c(t)+e(t) \cos \left(\omega_{e} t+\alpha_{e}\right)\right]} \\
+\frac{\pi}{2 V_{\pi(d c)}} V_{1 d c}
\end{array}\right) e^{\frac{j \Delta \varphi}{2}}
$$

Usando (10) y (11) se obtiene:

$$
\begin{aligned}
& E_{\text {out }}(t)=\frac{E_{\text {in }}}{2} \cos \\
& \left(\begin{array}{l}
\left.\frac{\pi}{2 V_{\pi(R F)}}\left[\begin{array}{l}
A_{1} V_{B B}(t)+V_{d c B B}+ \\
A_{2} V_{D R F}(t) \cos \left(\omega_{e} t+\phi_{e}\right)
\end{array}\right]+\right) e^{\frac{j \Delta \varphi}{2}} \\
\frac{\pi}{2 V_{\pi(d c)}} V_{1 d c}
\end{array}\right)
\end{aligned}
$$

Finalmente se obtiene la expresión en términos de los índices de modulación tanto de la señal de radiofrecuencia como de banda base.

$$
E_{\text {out }}(t)=\frac{E_{\text {in }}}{2} \cos \left(\begin{array}{l}
\frac{\pi}{2 V_{\pi(R F)}} V_{d c B B}+ \\
\frac{\pi}{2} m_{B B} V_{B B}(t)+ \\
\frac{\pi}{2} m_{R F} V_{D F R}(t) \\
\cos \left(\omega_{e} t+\phi_{e}\right)+\frac{\pi}{2 V_{\pi(d c)}} V_{1 d c}
\end{array}\right) e^{\frac{j \Delta \varphi}{2}}
$$

Donde $m_{B B}$ es el índice de modulación de la señal banda base y $m_{R F}$ es el índice de modulación de la señal de radiofrecuencia.

\section{RESULTADOS Y DISCUSIÓN}

El esquema de generación de señales para sistemas RoF usando combinación eléctrica de banda base y radiofrecuencia se modeló usando el software de simulación de sistemas ópticos VPITransmissionMaker, en el que la función de campo obtenida en (15), se evaluó para diferentes valores de índice de modulación de banda base y de RF. La evaluación de la calidad de la señal generada se realiza mediante el factor de calidad $Q$, el que se define como:

$$
Q=\frac{m_{1}-m_{0}}{\sigma_{1}+\sigma_{0}}
$$

Donde $m_{i} y \sigma_{i}$ representan la potencia media y desviación típica de potencia respectivamente del bit $i$ en el instante de decisión en el receptor. En este contexto, la amplitud de las señales de banda base y radiofrecuencia se varió entre $1,25 \mathrm{~V}$ y $6,25 \mathrm{~V}$ para conseguir índices de modulación de 0,25 a 1,25. La tasa de error de bit (BER) definida en función de $Q$ está dada por:

$$
B E R=\frac{1}{2} \operatorname{erfc}\left(\frac{Q}{\sqrt{2}}\right)
$$

Siendo erfc la función de error complementaria. A continuación se muestran un conjunto de figuras que sintetizan el comportamiento general del sistema en relación con el factor de calidad de las señales, permitiendo determinar las prestaciones del mismo para unas combinaciones de índices de modulación de banda base y RF determinadas. La Figura 4 muestra un comportamiento creciente del factor de calidad $Q$ de la señal de banda base con el incremento de su índice de modulación y una degradación con el aumento del índice de modulación de la señal de RF. También se observa un comportamiento adecuado para las señales de banda base cuando los índices de modulación de RF son inferiores a 0,75 , obteniendo valores de $\mathrm{Q} \geq 10,66$, equivalente a un valor de $\mathrm{BER}$ $\leq 7,8 \times 10^{-27}$, según (17). Para valores superiores del índice de modulación de la señal de RF, la señal de banda base se degrada considerablemente. Por ejemplo, si $m_{R F}=1, Q_{B B} \approx 2,60$, es decir, BER $\approx 4,6 \times 10^{-3}$.

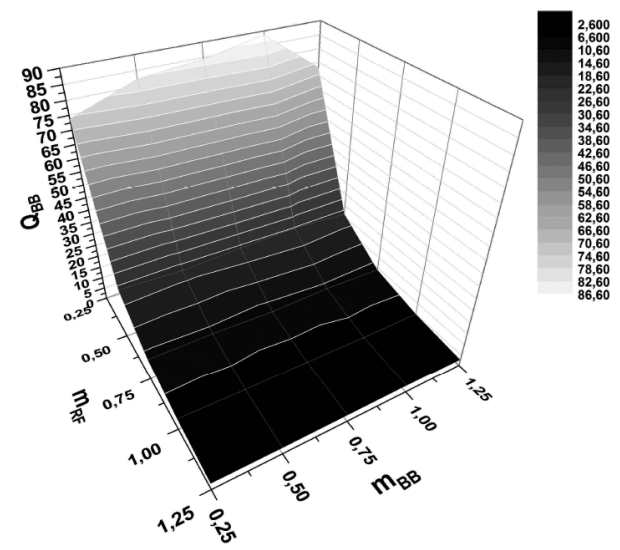

Figura 4. Comportamiento del factor de calidad de la señal RoF generada para la componente de banda base.

De otra parte, la Figura 5 muestra el comportamiento del factor de calidad de la señal de RF para diferentes valores del índice de modulación de la señal de banda base. La figura muestra un comportamiento 


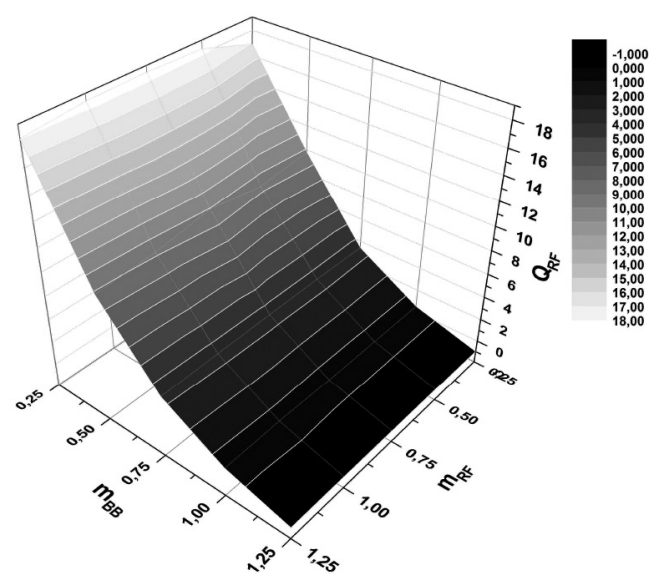

Figura 5. Comportamiento del factor de calidad de la señal de RoF para la componente de RF.

lineal del factor de calidad $Q$ de la señal de RF con el aumento de su índice de modulación para cualquier valor de índice de modulación de banda base. Este comportamiento se debe a que la señal de RF usará el rango dinámico disponible en el modulador en función del índice de modulación de la señal de banda base, a menor índice de modulación de banda base mayor es el rango dinámico disponible para la señal de RF, por lo tanto, $Q_{R F}$ aumenta cuando $m_{B B}$ disminuye. Los puntos óptimos de operación para se obtienen para $m_{B B} \leq 0,5$, consiguiendo valores de $Q_{R F} \geq 6$. Por ejemplo, para $m_{B B}=0,6, Q_{R F} \approx 8$, cuyo valor equivale a un BER de aproximadamente $5 \times 10^{16}$. La determinación de puntos adecuados de trabajo para el esquema en cuestión con las condiciones dadas se consigue a través de la figura de mérito, la que se obtiene realizando el producto punto a punto de las Figuras 4 y 5 . El resultado normalizado conseguido se muestra en la Figura 6 en donde se observa que el punto óptimo de trabajo se obtiene para la combinación de los índices de modulación $m_{B B}<0,25$ y $m_{R F}<0,25$. Del mismo modo, puntos adecuados de trabajo para $m_{B B}<0,75$ y $m_{R F}<0,5$ y puntos de trabajo deficientes en el resto de las combinaciones donde es imposible recuperar la información de alguna de las dos señales.

\section{CONCLUSIONES}

Este artículo presentó un esquema de generación de señales para sistemas de radio sobre fibra en donde la combinación de las señales de banda base

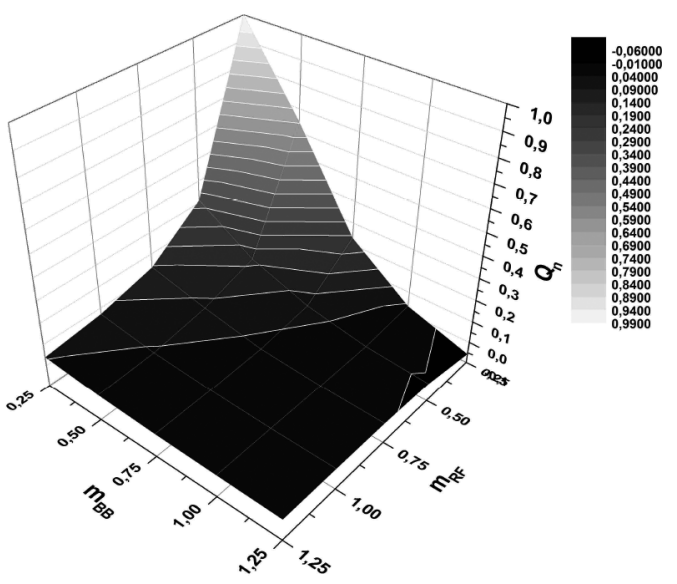

Figura 6. Figura de mérito para generación de señales RoF basado en combinación eléctrica de las componentes de banda base y RF.

y radio frecuencia se realizó en el dominio eléctrico y cuya señal resultante se utilizó como entrada a un brazo de la configuración interferométrica de un modulador electro-óptico tipo Mach-Zehnder. En este contexto, las señales en banda base se transmiten en la portadora óptica y las señales de radiofrecuencia se transportan como subportadoras de la portadora óptica en una frecuencia de la banda de microondas. Este paradigma de transmisión de señales es la base fundamental para las redes de nueva generación ya que permite establecer estrategias de convergencia entre redes fijas y redes inalámbricas i.e. redes móviles celulares que distribuyen sus portadoras entre diferentes estaciones base usando recursos físicos de una red FTTH.

Con base en la función de transferencia de la configuración interferométrica de modulador tipo $\mathrm{MZ}$, se derivaron las expresiones de campo eléctrico a la salida del sistema, en función de los índices de modulación, para una señal de entrada combinada de banda base y radiofrecuencia. Mediante variación de los índices de modulación, de dichas señales en la función de campo, se modeló el comportamiento del sistema y se encontraron los valores óptimos para los índices de modulación de la señal de banda base y radiofrecuencia. Con este procedimiento se encontró que los índices de modulación de ambas señales tienen una sólida dependencia entre sí, reflejándose dicho comportamiento en el factor de calidad de las señales, y que 
presenta un mínimo de penalización, cuando el índice de modulación de la señal de banda base $m_{B B}=m_{R F}=0,25$ consiguiendo valores de $Q_{R F} \mathrm{y}$ $Q_{B B} \geq 6$. Del mismo modo, se encontraron puntos adecuados de operación en donde la penalización, debida a la competencia por la utilización del rango dinámico del modulador no es significativa para $m_{B B}<0,75$ y $m_{R F}<0,5$ obteniéndose valores de $Q_{R F}$ y $m_{R F} \approx 6$. El resto de combinaciones penalizan de forma importante la calidad de las señales generadas.

\section{AGRADECIMIENTOS}

Los autores agradecen a la Universidad Distrital Francisco José de Caldas por apoyar la realización de este artículo.

\section{REFERENCIAS}

[1] Cisco, "Cisco Visual Networking Index: Forecast and Methodology 2013-2018". Cisco. Fecha de consulta: 17/07/2014. URL: http://www.cisco.com/en/US/solutions/ collateral/ns341/ns525/ns537/ns705/ns827/ white_paper_c11-481360.pdf.

[2] M. Marciniak. "Towards Broadband Global Optical and Wireless Networking". $11^{\text {th }}$ Management Committee Meeting of COST Action 273, Towards Mobile Broadband Multimedia Networks. Duisburg, Germany. Sept. 2004.

[3] J. Zhensheng, Y. Jianjun, E. Georgios and C. Gee-Kung. "Key Enabling Technologies for Optical-Wireless Networks: Optical Millimeter-Wave Generation, Wavelength Reuse, and Architecture". IEEE J. of Lightwave Technol. Vol. $25 \mathrm{~N}^{\circ} 11$, pp. 3452347. Nov. 2007. ISSN: 0733-8724. DOI: 10.1109/JLT.2007.909201.

[4] C. Gee-Kung, Y. Jianjun and J. Zhensheng. "Architectures and Enabling Technologies for Super-Broadband Radio-over-Fiber Optical-Wireless Access Networks". IEEE International Topical Meeting on Microwave Photonics. Victoria, Canada. Oct. 2007.

[5] L. Chun-Ting, C. Jason, P. Peng-Chun, P. Cheng-Feng, P. Wei-Ren, C. Bi-Shiou and C. Sien. "Hybrid Optical Access Network Integrating Fiber-to-the-Home and Radio-
Over-Fiber Systems". IEEE Photon Technol. Lett. Vol. $19 \mathrm{~N}^{\circ}$ 8, pp. 610-612. Apr. 2007. ISSN: 1041-1135. DOI: $10.1109 /$ LPT.2007.894326.

[6] D. Castleford, A. Nirmalathas, D. Novak and R. Tucker. "Optical crosstalk in fiber-radio WDM networks". IEEE Trans. Microw. Theory Tech. Vol. $49 \mathrm{~N}^{\circ} 10$, pp. 20302035. Oct. 2001. ISSN: 0018-9480. DOI: 10.1109/22.954826.

[7] H. Mizuguti, T. Okuno, S. Komaki and N. Morinaga. "Performance analysis of optical fiber link for microcellular mobile communication systems". IEICE Trans. Electron. E series. Vol. $76 \mathrm{~N}^{\circ} 2$, pp. 271-278. Feb. 1993. ISSN: 0916-8516.

[8] W. Way. "Optical fiber-based microcellular systems: An overview". IEICE Trans. Commun. Vol. 76 N $^{\circ}$ 9, pp. 1091-1102. Sept. 1993. ISSN: 0916-8516.

[9] C. Cox. "High-performance fiber-optic links for microwave applications". IEEE MTT-S International Microw. Symposium. Atlanta, USA. Jun. 1993.

[10] E. Ackerman, S. Wanuga, D. Kasemset, A. Daryoush and N. Samant. "Maximum dynamic range operation of a microwave external modulation fiber-optic link". IEEE Trans. Microw. Theory Tech. Vol. $41 \mathrm{~N}^{\circ} 8$, pp. 1299-1306. Aug. 1993. ISSN: 0018-9480. DOI: $10.1109 / 22.241670$.

[11] D. Sabido and L. Kazovsky, "Dynamic range of optically amplified RF optical links". IEEE Trans. Microw. Theory Tech. Vol. 49 $\mathrm{N}^{\circ} 10$, pp. 1950-1955. Oct. 2001. ISSN: 0018-9480. DOI: 10.1109/22.954813.

[12] R. Sabella. "Performance analysis of wireless broadband systems employing optical fiber links”. IEEE Trans. Commun. Vol. $47 \mathrm{~N}^{\circ} 5$, pp. 715-721. May. 2001. ISSN: 0090-6778. DOI: $10.1109 / 26.768765$.

[13] T. Kurniawan, A. Nirmalathas, C. Lim, D. Novak and R. Waterhouse. "Performance Analysis of Optimized Millimeter-Wave Fiber Radio Links". IEEE Trans. Microw. Theory Tech. Vol. $54 \mathrm{~N}^{\mathrm{o}}$ 2, pp. 921-928. Feb. 2006. ISSN: 0018-9480. DOI: 10.1109/ TMTT.2005.863047.

[14] C. Lim, M. Attygalle, A. Nirmalathas, D. Novak and R. Waterhouse. "Analysis of Optical Carrier-to-Sideband Ratio for 
Improving Transmission Performance in Fiber-Radio Links". IEEE Trans. Microw. Theory Tech. Vol. $54 \mathrm{~N}^{\circ}$ 5, pp. 2181-2187. May. 2006. ISSN: 0018-9480. DOI: 10.1109/ TMTT.2006.872809.

[15] A. Karim and J. Devenport. "Noise figure reduction in externally modulated analog fiber-optic links". IEEE Photon. Technol. Lett. Vol. $19 \mathrm{~N}^{\circ}$ 5, pp. 312-314. Mar. 2007. ISSN: 1041-1135. DOI: 10.1109/LPT.2007.891591.

[16] H. Zongjie, X. Zhang, Z. Shilie, J. Xiaofeng and C. Hao. "Any bias point control of mach-zehnder electrooptic modulator and its applications in optimization of radioover-fiber links". IEEE International Topical Meeting on Microwave Photonics. Singapore, Singapore. Oct. 2011.

[17] K. Prabu, K, S. Bose and D.S. Kumar. "Analysis of optical modulators for Radio over Free Space Optical Communication systems and Radio over Fiber systems". IEEE India Conference INDICON. Kochi, India. Dec. 2012.

[18] H. R. Cho, M. J. Shin, S. H. Han and J. W. Wu. "Mach-Zehnder interferometer measurement of the Pockels effect in a poled polymer film with a coplanar electrode structure". Applied Physics Lett. Vol. 69 No 25, pp. 3788-3790. Oct. 1996. ISSN: 0003-6951. DOI: 10.1063/1.116999.
[19] E. Yamada, Y. Shibata, K. Watanabe, T. Yasui, A. Ohki, H. Mawatari, S, Kanazawa, R. Iga and H. Ishii. "Demonstration of $50 \mathrm{Gbit} / \mathrm{s}$ 16QAM signal generation by novel 16QAM generation method using a dual-drive InP Mach-Zehnder modulator". Optical Fiber Communication Conference. Los Angeles, USA. Mar. 2011.

[20] K. Ho. "Generation of arbitrary quadrature signals using one dual drive modulator". IEEE J. Lightwave Technol. Vol. $23 \mathrm{~N}^{\circ} 2$, pp. 764-770. Feb. 2005. ISSN: 0733-8724. DOI: 10.1109/JLT.2004.838855.

[21] N. Kikuchi, E. Yamada, Y. Shibata and H. Ishii. "High-Speed InP-Based Mach-Zehnder Modulator for Advanced Modulation Formats". IEEE Compound Semiconductor Integrated Circuit Symposium. La Jolla, USA. Oct. 2012.

[22] R. Kaiser, K. Velthaus, T. Brast, M. Gruner, M. Hamacher, D. Hoffmann and M. Schell. "Medium and large scale Mach-Zehnder modulator ICs on InP for fabrication of advanced transmitters". International Conference on Transparent Optical Networks. Coventry, UK. Jul. 2012.

[23] G. P. Agrawal. "Fiber-Optic Communication Systems". John Wiley \& Sons. Third Edition. New York, USA. 2002. ISBN: 0-471-21571-6. 